\title{
Risk Factors for Chronic Kidney Disease in Pediatric Patients with Epilepsy
}

\author{
Jun Pak, MD, Jung Hye Byeon, MD, Chung Hyuk Yim, MD, Baik-Lin Eun, MD \\ Department of Pediatrics, Korea University College of Medicine, Seoul, Korea
}

Received: September 11, 2020

Revised: October 23, 2020

Accepted: October 26, 2020

Corresponding author:

Baik-Lin Eun, MD

Department of Pediatrics, Korea

University Guro Hospital, Korea

University College of Medicine, 148

Gurodong-ro, Guro-gu, Seoul

08308, Korea

Tel: +82-2-2626-1229

Fax: +82-2-2626-1249

E-mail: bleun@korea.ac.kr
Purpose: The aim of the treatment of epileptic seizures is to achieve a seizure-free state without adverse effects. The mainstays of seizure treatment are anticonvulsant medication, diet therapy, and surgery. Antiepileptic drugs and a ketogenic diet are associated with various types of medical adverse effects, including chronic kidney disease (CKD). We aimed to identify the characteristics of pediatric epilepsy patients who developed CKD and to determine the possible mechanisms. Methods: We included 816 patients who underwent medical treatment for epilepsy and were followed-up for at least 3 years at a tertiary hospital. The patients were divided into CKD and non-CKD groups. The data were assessed using a multivariate Cox proportional hazards model to identify the factors associated with CKD among patients undergoing epilepsy treatment.

Results: Initial high serum creatinine levels (hazard ratio [HR], 13.927; $P=0.010$ ), microscopic hematuria on initial urinalysis $(H R, 10.047 ; P=0.001)$, developmental delay $(H R, 11.929 ; P=0.000)$, and interictal epileptiform discharges on initial electroencephalography (generalized interictal epileptiform discharges: $H R$, 38.395, $P=0.003$; focal interictal epileptiform discharges: $H R$, 19.252, $P=0.006$ ) were associated with increased CKD risk.

Conclusion: CKD was more likely to develop in patients who presented with initial kidney dysfunction and developmental delay, and was related to epilepsy itself. The abovementioned factors may increase CKD risk through decreased brain function, which may lead to decreased activity and, hence, to relatively poor hygiene and voiding function. Moreover, patients with pre-existing kidney disease were more vulnerable to CKD development.

Keywords: Epilepsy; Renal insufficiency, chronic; Anticonvulsants; Risk; Child

\section{Introduction}

Epilepsy is a brain disorder which implies an enduring predisposition to unprovoked seizures $[1,2]$. The main focus of epilepsy treatment is satisfactory seizure control with minimal adverse effects [3]. Epilepsy treatments in children and adolescents are usually prescribed for at least 2 years (and for life-time in some cases), for which adverse effect control is of utmost importance $[2,4]$.
Patients undergoing long-term treatment with antiepileptic drugs (AEDs) may develop other diseases, as well as adverse effects from the drugs, which may limit AED usage $[4,5]$. Several AEDs undergo hepatic metabolism and are excreted via urine. Lamotrigine, phenobarbital, and lacosamide, which are often used in clinical practice, are examples of this pharmacokinetics. On the other hand, levetiramcetam and topiramate remain unchanged before being excreted via urine. Long-term use of these drugs can

Copyright (C) 2021 Korean Child Neurology Society

This is an Open Access article distributed under the terms of the Creative Commons Attribution Non-Commercial License (http://creativecommons.org/licenses/by-nc/4.0/) which permits unrestricted non-commercial use, distribution, and reproduction in any medium, provided the original work is properly cited. 
cause side effects on the kidneys. Some common adverse effects include nephrotoxicity, calciuria, and other events related to the kidneys $[3,5]$. However, and despite the abovementioned, only a few patients with long-term AED treatment develop chronic kidney disease (CKD).

In this paper, we investigated the characteristics of epilepsy patients who developed CKD. Through this study, we aimed to identify risk factors for $\mathrm{CKD}$ and analyze which factors should be considered in the treatment plan of patients with these characteristics.

\section{Materials and Methods}

\section{Patients}

A total of 816 patients who underwent medical treatment for epilepsy, including anticonvulsant medication, diet therapy, or surgery, and who were followed up for $>3$ years from 2016 to 2019 at the Korea University Guro Hospital were included in this study.

\section{Group definition}

The patients were divided into two groups: $\mathrm{CKD}$ and non-CKD. CKD was defined as decreased kidney function shown by glomerular filtration rate (GFR) of $<60 \mathrm{~mL} / \mathrm{min} / 1.73 \mathrm{~m}^{2}$, or the presence of markers of kidney damage, or both, of at least 3 months of duration regardless of underlying cause [6]. The markers of kidney damage considered for CKD diagnosis were urinary sediment abnormality, abnormal histological findings, and structural abnormalities detected by imaging $[6,7]$. The process of group classification is briefly explained in a flow diagram (Fig. 1).

\section{Pharmacological therapy}

Pharmacological treatment was usually initiated after epilepsy diagnosis and report of recurrent unprovoked seizures. The AED dose was gradually increased until the lowest effective maintenance dose to minimize adverse effects. If seizures persisted despite an AED up-titration to the maximum tolerated dose, the AED was switched to an alternative AED monotherapy. If two or three sequential monotherapies failed, polytherapy was offered. Polytherapy was considered earlier when prognostic factors indicated difficulty of treatment by monotherapy, considering the severity of the epilepsy. The cumulative treatment time of the administered AED was investigated to analyze its association with CKD.

\section{Ketogenic diet}

Despite the lack of a well-defined mechanism of action, several reports suggest that a ketogenic diet reduces seizure frequency $[8,9]$. In our center, the ketogenic diet was used as a treatment for drug-resistant pediatric epilepsy. In particular, a ketogenic diet was prescribed in cases of intractable epilepsy with myoclonic seizures, atonic seizures, or mixed seizures, as is the case in Lennox-Gastaut syndrome (LGS). In these patients, the classical ketogenic diet, which is based on a 3:1 ratio and a 4:1 ratio of high fat to low carbohydrate and to protein, respectively, was used $[8,9]$.

\section{Statistical analysis}

Continuous data are presented as mean \pm standard deviation and were evaluated using an independent samples t-test. Categorical data are presented as percentages and were evaluated using the chisquare test (Table 1). The Cox proportional hazards model was used to compare the variables between CKD and non-CKD groups (Table 2). Data in Table 2 were investigated using univariate analysis. Finally, all data in Table 2 were analyzed using the adjusted multivariate Cox proportional hazards model for variables such as age, sex, follow-up duration, number of AEDs, ketogenic diet, each AED's treatment time, initial laboratory findings, electroencephalography (EEG) findings, initial magnetic resonance imaging (MRI) findings, and developmental delay. The risk difference was reported as odds ratios with $95 \%$ confidence interval (CI). All reported $P$ values are two-sided. All statistical analyses were performed using SPSS version 20 (IBM Co., Armonk, NY, USA). Results were considered statistically significant if $P<0.05$.

\section{Ethical approval}

The study procedures were approved and monitored by the Institutional Review Board of the Korea University Medical Center (IRB No. 2020GR0312) and were conducted in compliance with the Declaration of Helsinki and the Good Clinical Practice guidelines.

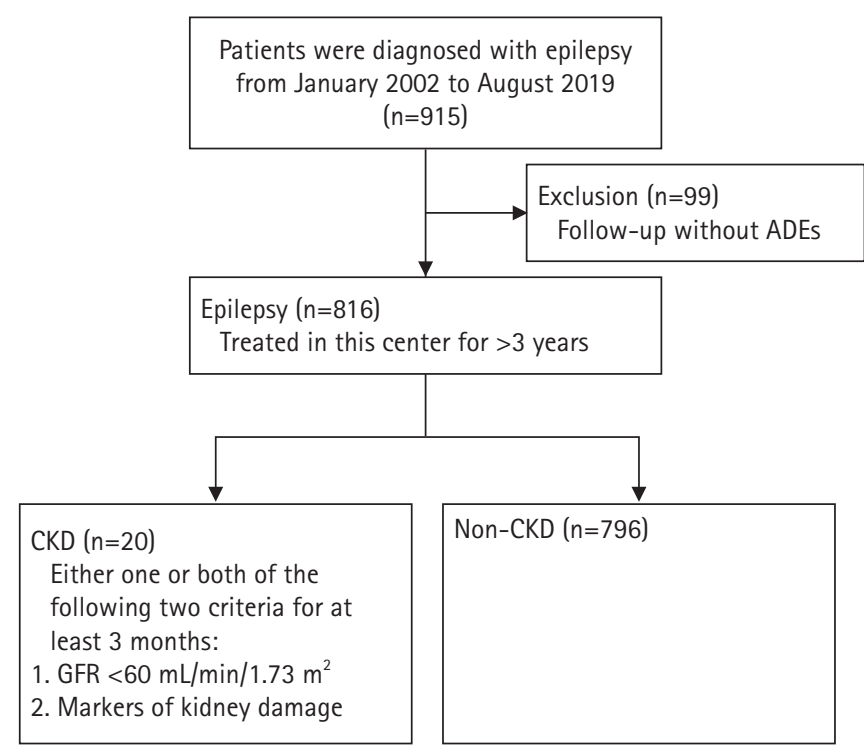

Fig. 1. Patient selection process. AED, antiepileptic drug; CKD, chronic kidney disease; GFR, glomerular filtration rate. 
Table 1. Baseline characteristics of the patients classified by CKD status

\begin{tabular}{|c|c|c|c|c|}
\hline Characteristic & Overall $(n=816)$ & Non-CKD $(n=796)$ & CKD $(n=20)$ & $P$ value \\
\hline Age (mo) & $14.97 \pm 6.55$ & $14.95 \pm 6.56$ & $15.95 \pm 6.27$ & $0.383^{\mathrm{a}}$ \\
\hline Male sex & 487 (59.68) & $473(59.42)$ & $14(70.00)$ & 0.341 \\
\hline Follow-up duration (mo) & $117.92 \pm 46.72$ & $117.93 \pm 46.74$ & $117.58 \pm 46.82$ & $0.898^{2}$ \\
\hline AED medications & $2.17 \pm 1.27$ & $2.15 \pm 1.27$ & $2.65 \pm 1.31$ & $0.055^{a}$ \\
\hline Ketogenic diet & $11(1.35)$ & $10(1.26)$ & $1(5.00)$ & $0.240^{b}$ \\
\hline \multicolumn{5}{|l|}{ AED medication period (mo) } \\
\hline Valproic acid & $48.13 \pm 62.98$ & $47.80 \pm 62.65$ & $61.40 \pm 75.34$ & $0.569^{\circ}$ \\
\hline Levetiracetam & $33.33 \pm 43.12$ & $33.04 \pm 43.00$ & $45.05 \pm 47.23$ & $0.087^{\circ}$ \\
\hline Oxcarbazepine & $30.35 \pm 47.28$ & $30.49 \pm 47.57$ & $24.80 \pm 34.40$ & $0.909^{2}$ \\
\hline Topiramate & $17.54 \pm 38.48$ & $17.49 \pm 38.52$ & $20.40 \pm 37.84$ & $0.675^{\circ}$ \\
\hline Phenobarbital & $6.56 \pm 24.21$ & $6.34 \pm 23.84$ & $15.25 \pm 35.86$ & $0.051^{\mathrm{a}}$ \\
\hline Phenytoin & $0.83 \pm 7.85$ & $0.84 \pm 7.94$ & $0.50 \pm 2.01$ & $0.177^{2}$ \\
\hline Lamotrigine & $20.63 \pm 46.50$ & $20.61 \pm 46.48$ & $21.40 \pm 48.36$ & $0.939^{\circ}$ \\
\hline Clobazam (BDZ) & $11.18 \pm 35.29$ & $10.79 \pm 34.60$ & $26.75 \pm 55.49$ & $0.101^{\mathrm{a}}$ \\
\hline Perampanel & $1.51 \pm 6.41$ & $1.48 \pm 6.35$ & $3.00 \pm 8.57$ & $0.016^{2}$ \\
\hline Vigabatrin & $4.38 \pm 18.56$ & $4.38 \pm 18.65$ & $4.20 \pm 15.09$ & $0.957^{\circ}$ \\
\hline \multicolumn{5}{|l|}{ Initial laboratory findings } \\
\hline BUN (mg/dL) & $11.71 \pm 3.66$ & $11.70 \pm 3.61$ & $12.08 \pm 5.38$ & $0.579^{2}$ \\
\hline Creatinine (mg/dL) & $0.47 \pm 0.19$ & $0.47 \pm 0.19$ & $0.55 \pm 0.33$ & $0.324^{2}$ \\
\hline Microscopic hematuria & $31(3.80)$ & 27 (3.39) & $4(20.00)$ & $0.005^{b}$ \\
\hline Dipstick proteinuria & $217(26.59)$ & $211(26.51)$ & $6(30.00)$ & 0.727 \\
\hline Inter-ictal epileptiform discharges on initial EEG & & & & 0.006 \\
\hline No inter-ictal epileptiform discharges & $367(44.98)$ & $365(45.85)$ & $2(10.00)$ & \\
\hline Generalized inter-ictal epileptiform discharges & $64(7.84)$ & $61(7.66)$ & $3(15.00)$ & \\
\hline Focal inter-ictal epileptiform discharges & 385 (47.18) & $370(46.48)$ & $15(75.00)$ & \\
\hline Abnormal background activity on initial EEG & $120(14.71)$ & $111(13.94)$ & $9(45.00)$ & $0.001^{b}$ \\
\hline Brain abnormality on initial MRI & 159 (19.49) & $151(18.97)$ & $8(40.00)$ & $0.039^{b}$ \\
\hline Kidney abnormality on initial US & $31(3.80)$ & $26(3.27)$ & $5(25.00)$ & $0.001^{b}$ \\
\hline Developmental delay & $100(12.25)$ & $88(11.06)$ & $12(60.00)$ & $0.000^{b}$ \\
\hline
\end{tabular}

Values are presented as mean \pm standard deviation or number $(\%)$.

CKD, chronic kidney disease; AED, antiepileptic drug; BUN, blood urea nitrogen; EEG, electroencephalography; MRI, magnetic resonance imaging; US, ultrasonography.

${ }^{a}$ Mann-Whitney U test; ${ }^{b}$ Fisher exact test.

Written informed consent by the patients was waived due to a retrospective nature of our study.

\section{Results}

\section{Patients' clinical characteristics at baseline}

Table 1 presents the descriptive baseline characteristics of the study sample. The mean age was $14.97 \pm 6.55$ years and females comprised 59.68\% of the included patient population. The follow-up period was $117.92 \pm 46.72$ months from the initial visit to our center. These patients were treated with $2.17 \pm 1.27 \mathrm{AEDs}$ during the follow-up period. All patients received AED treatment, and 11 (1.35\%) were prescribed ketogenic diet in addition. The details of each AED's treatment time is described in Table 1. Regarding labo- ratory findings, all patients underwent blood tests and urinalysis periodically during AED treatment to detect adverse effects. The blood urea nitrogen and the creatinine levels were within the normal range at the initial evaluation (blood urea nitrogen 11.71 \pm 3.66 ; creatinine $0.47 \pm 0.19)$. Thirty-one patients $(3.80 \%)$ had microscopic hematuria and 217 (26.59\%) had dipstick proteinuria at baseline. In the initial EEG test, 64 (7.84\%) and 385 patients (47.18\%) showed generalized and focal epileptiform discharges, respectively. Moreover, background activity was abnormal in 120 (14.71\%) patients. All patients in this study underwent brain MRI at diagnosis, and 159 (19.49\%) presented structural anomalies of the brain, including hippocampal sclerosis, ventriculomegaly, periventricular leukomalacia, and masses. Developmental delay was reported in 100 patients (12.25\%) at initial evaluation, and 31 
(3.80\%) presented abnormal findings on abdominal kidney ultrasonography.

There were significant differences in microscopic hematuria, interictal epileptiform discharges, abnormal background activity on initial EEG, brain abnormalities on initial MRI, kidney abnormalities on initial ultrasonography, developmental delay at baseline, and Perampanel treatment time between $\mathrm{CKD}$ and non-CKD groups. The treatment time of Perampanel was significantly different between the two groups in an independent samples t-test. Perampanel undergoes oxidation mediated by hepatic metabolism and is excreted via feces (48\%) and urine (22\%). It is well known that patients treated with perampanel are prone to urinary tract infection and hyponatremia $[10,11]$. In contrast, treatment time of other AEDs did not show statistical significance.

\section{Factors influencing CKD incidence among patients' initial characteristics and epilepsy therapy}

To assess the factors influencing kidney function, the multivariate Cox proportional hazards model was performed. All the parameters in Table 2 were candidate variables for this analysis. The univariate Cox proportional hazards model results shown in Table 2 indicated statistical significance for microscopic hematuria, interictal epileptiform discharges, abnormal background activity on initial EEG, brain abnormalities on initial MRI, kidney abnormality on initial ultrasonography, and developmental delay at baseline between the $\mathrm{CKD}$ and non-CKD groups. However, the multivariate Cox proportional hazards model using backward stepwise (likelihood ratio) selected four prognostic factors associated with an increased risk of CKD: serum creatinine (hazard ratio [HR], 13.927; 95\% CI, 1.857 to $104.455 ; P=0.010)$; microscopic hematuria on

Table 2. Results from the Cox proportional hazards models

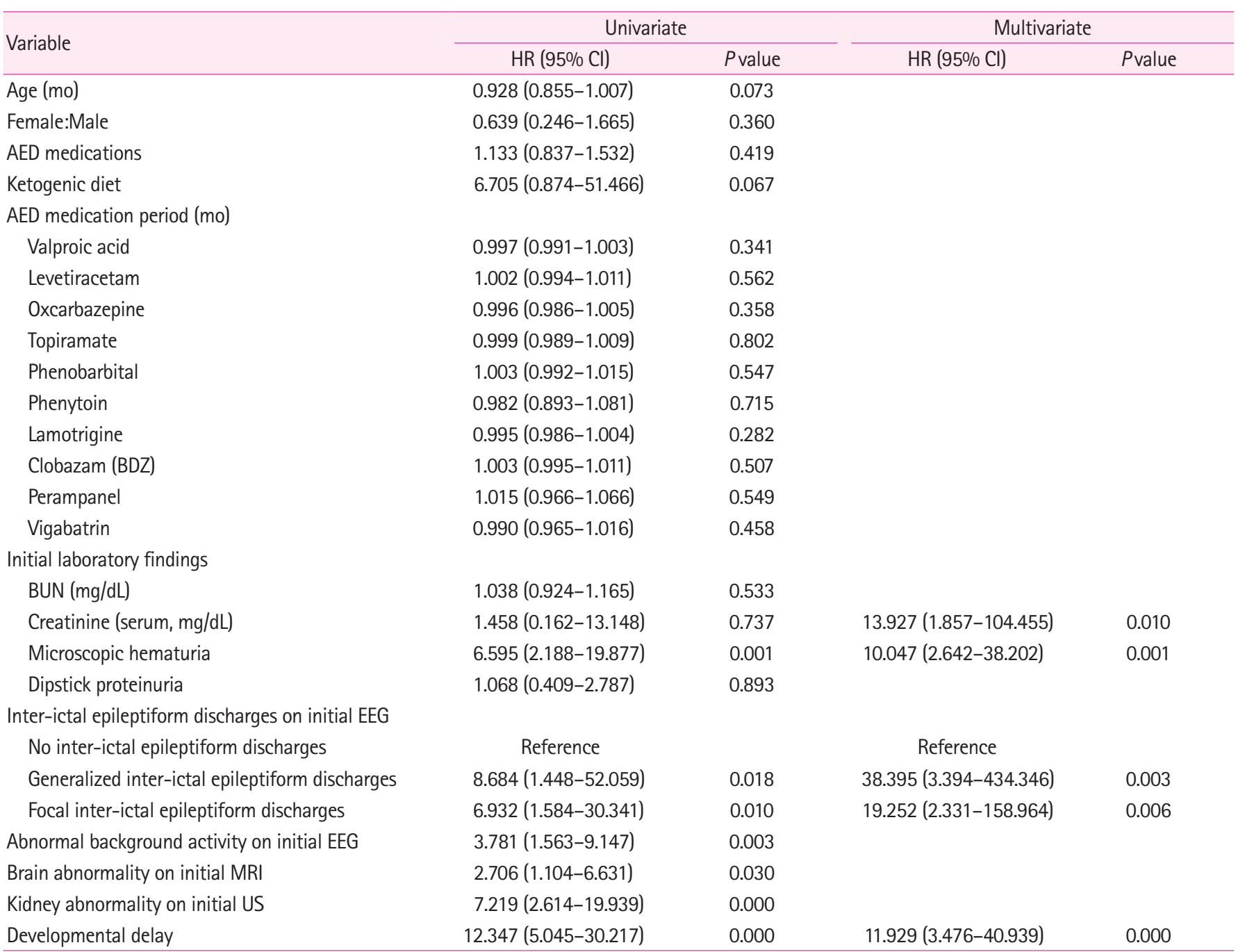

$\mathrm{HR}$, hazard ratio; $\mathrm{Cl}$, confidence interval; $\mathrm{AED}$, antiepileptic drug; BUN, blood urea nitrogen; EEG, electroencephalography; MRI, magnetic resonance imaging; US, ultrasonography. 
initial urinalysis ( $\mathrm{HR}, 10.047$; 95\% CI, 2.642 to 38.202; $P=0.001)$; developmental delay (HR, 11.929; 95\% CI, 3.476 to 40.939; $P=0.000$ ); and interictal epileptiform discharges on initial EEG (generalized interictal epileptiform discharges [HR, 38.395, 95\% CI, 3.394 to 434.346; $P=0.003$ ], focal interictal epileptiform discharges [HR, 19.252; 95\% CI, 2.331 to $158.964 ; P=0.006]$ ).

In Kaplan-Meier curves, which are widely used to estimate the survival function, the probability of CKD differed significantly according to the number of risk factors. The survival curve decreased rapidly as the number of significant risk factors increased (Fig. 2).

\section{Discussion}

In this study aimed at determining risk factors for $\mathrm{CKD}$ in pediatric epilepsy patients, we detected four significant risk factors, namely, initial creatinine, microscopic hematuria, interictal epileptiform discharges, developmental delay.

All patients with ongoing AED treatment underwent urinalysis to detect adverse effects such as nephrotoxicity, hypercalciuria, and nephrolithiasis. A statistical analysis was performed to confirm whether initial hematuria affects kidney function and the multivariate Cox proportional hazards model showed that microscopic hematuria was significantly different between the CKD group (four patients $[20.00 \%]$ ) and the non-CKD group (27 patients [3.39 $\%]$ ). Microscopic hematuria is a common clinical manifestation in children, adolescents, and young adults, with its incidence ranging from $0.18 \%$ to $16.1 \%$ according to previous studies [12-14]. Hematuria can either be an isolated finding unaccompanied by other urinary abnormalities or be accompanied by urinary abnormalities such as proteinuria, hypertension, and elevated serum creatinine level $[13,14]$. Persistent isolated microscopic hematuria can have either a glomerular or a non-glomerular origin, including several kidney disease entities [12,13]. In fact, among the 20 patients in the CKD group, four patients presented glomerulonephritis in the histopathologic examination. The four cases of glomerulonephritis were classified as Henoch-Schonlein nephritis (grade III), focal segmental endocapillary proliferative glomerulonephritis, focal segmental glomerulosclerosis, and minor glomerular change on renal histological examination. Additionally, four patients reported recurring urinary tract infections and nephrolithiasis. The causes of hematuria mentioned above increase the risk of CKD [14]. In other studies, $0.7 \%$ of patients with persistent isolated microscopic hematuria developed end-stage kidney disease compared with $0.04 \%$ in the control group, yielding an adjusted HR of 18.5 (95\% CI, 12.4 to 27.6 ) [14].

The multivariate Cox proportional hazard model result showed that increased serum creatinine levels significantly increased the risk of CKD (HR, 13.927; 95\% CI, 1.857 to 104.455 ; $P=0.010$ ). Because creatinine is excreted from the circulation mainly by renal filtration, it is strongly correlated with GFR [15-17]. An increase in serum creatinine reflects an impaired renal ability to remove waste products from the body [16]. The creatinine concentration increases progressively with decreased GFR, and its diagnostic performance has been shown to be good, even for the detection of minor deteriorations of the renal function $[15,16]$. Hence, creatinine is a strong predictor of $\mathrm{CKD}$ progression [16].

In this study on the prediction of risk factors of $\mathrm{CKD}$, the EEG results were analyzed by classifying them into different findings

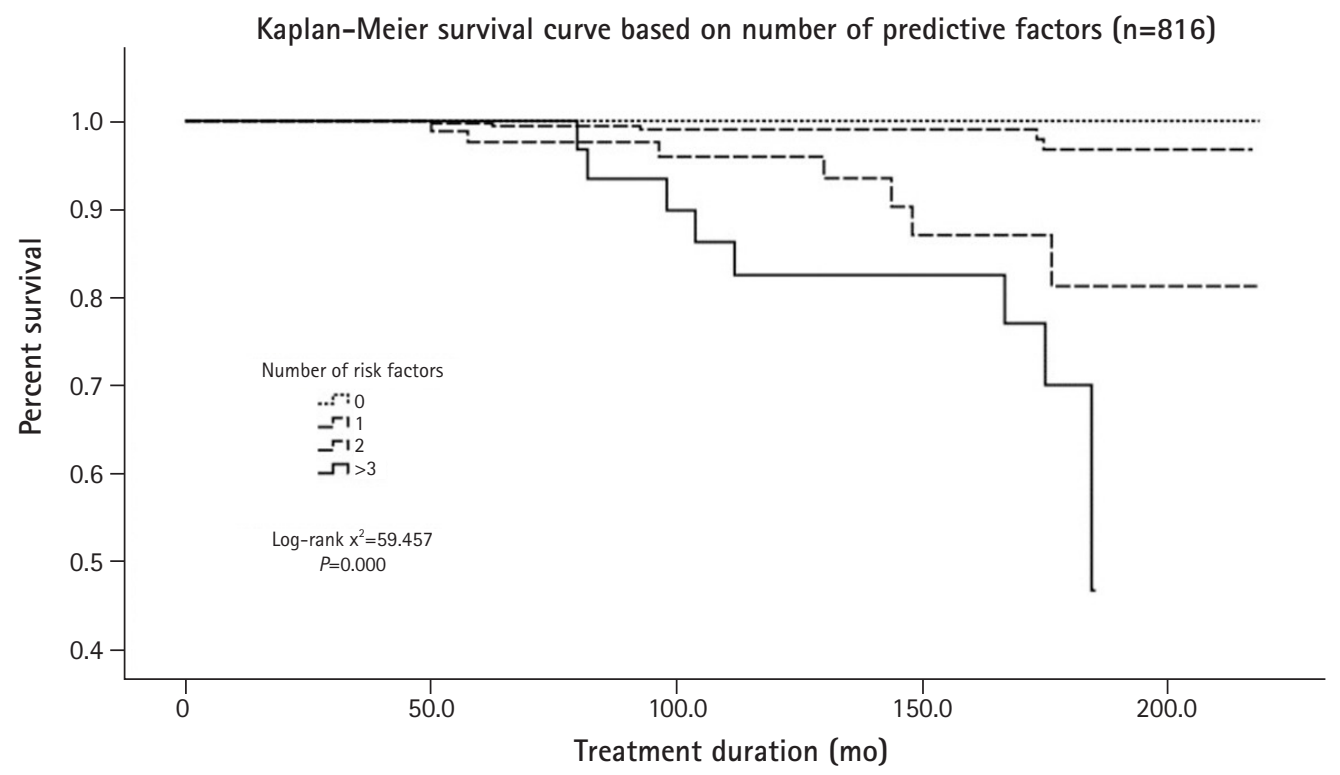

Fig. 2. In Kaplan-Meier curves, the probability of chronic kidney disease differed significantly according to the number of risk factors. 
rather than into a clinical diagnosis. Regarding the presence of interictal epileptiform discharges, the HR of generalized interictal epileptiform discharges was 38.395 , and that of focal interictal epileptiform discharges was 19.252 in the CKD group compared to the non-CKD group for initial interictal EEG findings. Patients with interictal epileptiform discharges on EEG tend to have clinical seizure events in contrast to those without. Recurrent clinical seizure events impair cognitive abilities and neurological development. Regarding background activity, when there was a disorganization, the $\mathrm{HR}$ was 4.201, indicating borderline significance. $\mathrm{Ab}$ normal background activity on the EEG includes a lack of the expected organization corresponding to age, multifocal spikes, and loss of normal physiological hallmarks, like sleep spindles [18-20]. These EEG findings are seen in most cases where the brain is structurally and functionally deteriorated, including in LGS, Rett syndrome, and West syndrome. In this study, seven patients with CKD who experienced intractable epilepsy (e.g., LGS, Rett syndrome, West syndrome, Devic syndrome, Wolf-Hirschhorn syndrome) were bedridden [18-20]. The comparison was not statistically significant; however, it is possible that abnormal background activity acts as a significant risk factor in larger patient groups considering its borderline significance. In general, only $29 \%$ to $55 \%$ of epilepsy patients have epileptiform abnormalities on a single routine outpatient EEG. Therefore, even if normal EEG findings are reported in the first examination, abnormal findings often appear in the second and third examinations. However, in the present study we only analyzed the initial EEG results to assess the risk of $\mathrm{CKD}$ at the initial planning of diagnosis and treatment.

In addition, $12(60 \%)$ patients in CKD group, including those with intractable epilepsy, had developmental delays. In such cases, the patients' brain dysfunction may not directly unable to have normal daily lives, it may lead to poor sanitation and urinary retention without voluntary urination [21]. These patients might be more vulnerable to nephrotoxicity induced by the long-term administration of AEDs. The patients' decreased voiding function may lead to residual urine. Among these patients, some developed urinary tract stones and recurrent urinary tract infections, which leads to acute kidney injury and affects renal function, and which can contribute to the development of CKD. In the present case, the statistically significant HR related to developmental delay was 11.929 .

In conclusion, the risk of CKD increases in cases of epilepsy with high creatinine and microscopic hematuria associated with kidney damage, and with restricted daily activity due to a decreased brain function. In this study on factors associated with an increased risk of $\mathrm{CKD}$, the abovementioned factors had statistically significant results while AED-related factors did not. In the long-term treat- ment of epilepsy patients, it is important to perform regular kidney function evaluations and to practice regular urine voiding to prevent the development of $\mathrm{CKD}$, in addition to following-up potential AED adverse effects.

This study has some limitations. The study was retrospective in nature, and the sample was not randomized. Moreover, the patients were prescribed different AEDs not based on the medical condition alone, but also for social reasons. In addition, it is possible that factors including AED history, ketogenic diet, and abdominal ultrasonography were not statistically significant owing to the relatively small number of patients in the CKD group. As shown in the study results, AEDs that lead to nephrotoxicity might act as risk factors for patients with vulnerability factors for CKD. Further study is need to validate the risk of $C K D$ generated by AEDs, ketogenic diet, EEG findings, brain function, and kidney factors in larger CKD patient groups.

\section{Conflicts of interest}

No potential conflict of interest relevant to this article was reported.

\section{ORCID}

Jun Pak, https://orcid.org/0000-0002-5634-6559

Baik-Lin Eun, https://orcid.org/0000-0001-8735-292X

\section{Author contribution}

Conceptualization: JP, CHY, and BLE. Data curation: BLE. Formal analysis: JP, JHB, and BLE. Methodology: JP. Writing-original draft: JP. Writing-review \& editing: JP, JHB, CHY, and BLE.

\section{Acknowledgements}

This work was supported by an Institute for Information \& Communications Technology Promotion (IITP) grant funded by the Korean government (MSIT) (No. 2018-0-00861).

\section{References}

1. Fisher RS, van Emde Boas W, Blume W, Elger C, Genton P, Lee P, et al. Epileptic seizures and epilepsy: definitions proposed by the International League Against Epilepsy (ILAE) and the International Bureau for Epilepsy (IBE). Epilepsia 2005;46:470-2.

2. Perucca P, Scheffer IE, Kiley M. The management of epilepsy in children and adults. Med J Aust 2018;208:226-33. 
3. Rogawski MA, Loscher W. The neurobiology of antiepileptic drugs. Nat Rev Neurosci 2004;5:553-64.

4. Ruiz-Gimenez J, Sanchez-Alvarez JC, Canadillas-Hidalgo F, Serrano-Castro PJ; Andalusian Epilepsy Society. Antiepileptic treatment in patients with epilepsy and other comorbidities. Seizure 2010;19:375-82.

5. Titoff V, Moury HN, Titoff IB, Kelly KM. Seizures, antiepileptic drugs, and CKD. Am J Kidney Dis 2019;73:90-101.

6. Paniagua-Sierra JR, Galvan-Plata ME. Chronic kidney disease. Rev Med Inst Mex Seguro Soc 2017;55:S116-7.

7. Kazancioglu R. Risk factors for chronic kidney disease: an update. Kidney Int Suppl (2011) 2013;3:368-71.

8. Lefevre F, Aronson N. Ketogenic diet for the treatment of refractory epilepsy in children: a systematic review of efficacy. Pediatrics 2000;105:E46.

9. Neal EG, Chaffe H, Schwartz RH, Lawson MS, Edwards N, Fitzsimmons G, et al. The ketogenic diet for the treatment of childhood epilepsy: a randomised controlled trial. Lancet Neurol 2008;7:500-6.

10. French JA, Krauss GL, Biton V, Squillacote D, Yang H, Laurenza A, et al. Adjunctive perampanel for refractory partial-onset seizures: randomized phase III study 304. Neurology 2012;79: 589-96.

11. Krauss GL, Serratosa JM, Villanueva V, Endziniene M, Hong Z, French J, et al. Randomized phase III study 306: adjunctive perampanel for refractory partial-onset seizures. Neurology 2012; 78:1408-15.

12. Vivante A, Afek A, Frenkel-Nir Y, Tzur D, Farfel A, Golan E, et al. Persistent asymptomatic isolated microscopic hematuria in
Israeli adolescents and young adults and risk for end-stage renal disease. JAMA 2011;306:729-36.

13. Chan D, Ong A, Schoenberg M. Microscopic hematuria. N Engl J Med 2003;349:1292-3.

14. Vivante A, Calderon-Margalit R, Skorecki K. Hematuria and risk for end-stage kidney disease. Curr Opin Nephrol Hypertens 2013;22:325-30.

15. Rule AD, Larson TS, Bergstralh EJ, Slezak JM, Jacobsen SJ, Cosio FG. Using serum creatinine to estimate glomerular filtration rate: accuracy in good health and in chronic kidney disease. Ann Intern Med 2004;141:929-37.

16. Spanaus KS, Kollerits B, Ritz E, Hersberger M, Kronenberg F, von Eckardstein A, et al. Serum creatinine, cystatin C, and beta-trace protein in diagnostic staging and predicting progression of primary nondiabetic chronic kidney disease. Clin Chem 2010;56:740-9.

17. Wyss M, Kaddurah-Daouk R. Creatine and creatinine metabolism. Physiol Rev 2000;80:1107-213.

18. Engel J Jr. A practical guide for routine EEG studies in epilepsy.J Clin Neurophysiol 1984;1:109-42.

19. Noachtar S, Remi J. The role of EEG in epilepsy: a critical review. Epilepsy Behav 2009; 15:22-33.

20. Gmehlin D, Thomas C, Weisbrod M, Walther S, Pfuller U, Resch F, et al. Individual analysis of EEG background-activity within school age: impact of age and sex within a longitudinal data set. Int J Dev Neurosci 2011;29:163-70.

21. Penna FJ, Elder JS. CKD and bladder problems in children. Adv Chronic Kidney Dis 2011;18:362-9. 